\title{
Desarrollo teórico y evidencia empírica de la política de dividendos: una revisión de literatura en Iberoamérica *
}

\author{
Theorical Development and Empirical Evidence of Dividend Policy: A Review of \\ Ibero-American Literature
}

\author{
Albeiro Aguirre-Ríos \\ Magíster en Economía, Corporación Universitaria Americana, \\ Medellín-Colombia, aaguirre@americana.edu.co \\ Jovany Sepúlveda-Aguirre \\ Magíster en Gestión de la Innovación Tecnológica, Corporación Universitaria Americana, \\ Medellín-Colombia, jasepulveda@americana.edu.co \\ Uvenny Quirama-Estrada \\ Magíster en Administración, Corporación Universitaria Americana, \\ Medellín-Colombia, uquirama@americana.edu.co \\ Luis Fernando Garcés-Giraldo \\ Doctor en Filosofía, Corporación Universitaria Americana, \\ Medellín-Colombia, Igarces@americana.edu.co
}

Cómo citar / How to cite

Aguirre-Ríos, A.; Sepúlveda-Aguirre, J.; Quirama-Estrada, U.; Garcés-Giraldo, L. F. (2021). Desarrollo teórico y evidencia empírica de la política de dividendos: una revisión de literatura en Iberoamérica. Revista CEA, v. 7, n. 13, e1595. https://doi.org/10.22430/24223182.1595

Recibido: 22 de junio de 2020

Aceptado: 30 de septiembre de 2020

\section{Resumen}

La política de dividendos en una organización está relacionada con las decisiones de inversión y endeudamiento, claves para su permanencia en el tiempo. El objetivo de este documento es examinar la evidencia empírica y desarrollo teórico en políticas de dividendos a partir de una revisión de literatura. La metodología de la revisión fue la búsqueda en las bases de datos Scopus, Ebsco, Redalyc y Google Académico, a partir de las palabras clave y teniendo en cuenta estudios realizados en Iberoamérica. El principal hallazgo obtenido es que la mayoría de los estudios están a favor del

\footnotetext{
* Este artículo se deriva del proyecto titulado «Determinantes de la política de dividendos: Análisis de datos de panel en empresas industriales que cotizan en la Bolsa de Valores de Colombia (2009-2014)» y ha sido financiado con recursos propios.
} 
enfoque de la relevancia de la política de dividendos mediante el uso de modelos cuantitativos enfocados a identificar sus determinantes. Se concluye que la estructura teórica del pago de dividendos se fortaleció con el estudio de las imperfecciones de los mercados financieros y que las teorías más contrastadas en la región corresponden a las del enfoque de la relevancia, en particular, costos de agencia y valor informativo de los dividendos. Los países donde se concentran la mayoría de las investigaciones son Brasil, Chile y España.

Palabras clave: dividendo, política de dividendos-Iberoamérica, revisión de la literatura.

\title{
Clasificación JEL: G35.
}

\begin{abstract}
The dividend policy of an organization is related to their investment and credit decisions, which are key for their permanence over time. This paper aims to review the available literature on empirical evidence and theoretical developments of dividend policy. The methodology implemented here was a search in Scopus, Ebsco, Redalyc, and Google Scholar with specific keywords and limited to studies conducted in Ibero-America. The first finding is that most studies support the dividend relevance theory by using quantitative models aimed at identifying its determinants. We conclude that the theoretical structure of dividend payment was strengthened by research into the imperfections of financial markets, and the theories most commonly addressed in this region are those in the relevance approach; in particular, agency costs and the informational value of dividends. Finally, Brazil, Chile, and Spain concentrate most studies in this field in Ibero-America.
\end{abstract}

Keywords: Dividend, dividend policy-Iberoamerica, literature review.

\section{JEL classification: G35.}

\section{INTRODUCCIÓN}

El estudio de la política de dividendos inicia con las disertaciones de Lintner (1956), seguido de Miller y Modigliani (1961) sobre su relevancia e irrelevancia, respectivamente; posteriormente, otros autores incluyeron nuevos elementos a la discusión, como los costos de transacción por parte de los costos de agencia, la asimetría de la información por el valor informativo de los dividendos por el efecto clientela estudiado, configurando una amplia categoría de estudio en el mundo de las finanzas (Higgins, 1972; Rozeff, 1982; Easterbrook, 1984; Jensen, 1986; Akerlof, 1970; Bhattacharya, 1979; Miller \& Rock, 1985; Ross, 1977; John \& Willians, 1985; Kumar, 1988; Elton \& Gruber, 1970; Bajaj \& Vijh, 1990).

A nivel empírico, la mayoría de estudios sobre la aplicación de la política de dividendos concluyen que es relevante, como los realizados por La Porta et al., (2000); Smith (2011); Chahyadi y Salas (2012); Deshmukh et al., (2013) y Baker y Weigand (2015); es decir, que existe una relación directa entre el pago de dividendos y el valor de la firma, por lo que identificar los determinantes que inciden en el pago de éstos ayudaría a entender las razones y circunstancias bajo las cuales las empresas diseñan su política de dividendos y, por otra parte, a identificar elementos de juicio para los inversionistas del mercado bursátil. 
En Iberoamérica se han realizado estudios sobre este tema principalmente en Brasil, Chile y España; en Colombia, Usma Patiño y Ramírez Arango (2010) desarrollaron un estudio donde se analiza al gobierno corporativo como único determinante de la política de dividendos, concluyendo que existe una relación directa como lo esperaban estos autores; Álvarez Agudelo (2013), analiza el impacto de la política de distribución de dividendos sobre la rentabilidad del inversionista entre los años 2008 y 2013 en el mercado colombiano, concluyendo que las empresas que reparten mayor dividendo han presentado un crecimiento constante en sus utilidades netas, las compañías nacientes tienen un desempeño superior en el ritmo de crecimiento de sus ganancias y que los accionistas minoritarios prefieren las acciones que paguen mayores dividendos.

\section{MARCO TEÓRICO}

\section{La política de dividendos}

Una definición de dividendo que reúne los elementos básicos la presentan Ross et al., (2010) «es un pago efectuado de las utilidades de una empresa a sus propietarios, en acciones o en efectivo» ( $p$. 547). La política de dividendos, según Berk \& DeMarzo, (2010), es la alternativa que eligen las empresas para remunerar a los accionistas.

Existe un costo de oportunidad en el pago de dividendos, por lo cual definir la cuantía de utilidades que se entregan por este concepto a sus accionistas tiene gran influencia en el futuro de la empresa (Davaadorj, 2019), en otras palabras:

La política de dividendos forma parte de las decisiones de financiación de la empresa, puesto que cualquier dinero pagado de dividendos deberá ser financiado de alguna manera, ya sea con un nuevo endeudamiento o con una nueva ampliación del capital (Mascareñas, 2011, p.1).

En general se presentan tres tipos de políticas de dividendos. Siguiendo a Higuerey (2014), la primera es cuando la firma establece una razón de pago constante de dividendos sobre las utilidades; la segunda es una política de dividendos regulares, en la cual se establece un dividendo fijo en unidades monetarias que puede modificarse en presencia de un aumento sostenible de las utilidades; y el tercero es una política de dividendos bajos, regulares y extraordinarios, donde se garantiza un pago bajo y constante para los inversionistas con la condición de tener dividendos extraordinarios en circunstancias especiales. En la Tabla 1, se enfrentan dos posturas contrarias al pago de dividendos que involucran a inversionistas y administradores de las empresas emisoras de acciones.

La discusión en torno a las políticas de dividendos comienza con Lintner (1956) y Gordon (1959) argumentando que existe una relación directa entre el pago de dividendos y el valor de la firma, así como mediante las tendencias de crecimiento de la economía en el largo plazo, lo cual se conoce como la «teoría de la relevancia de los dividendos»; posteriormente, Miller y Modigliani (1961) plantearon que la política de dividendos es irrelevante en el escenario de mercados de capitales perfectos, asumiendo que el valor de la firma es determinado por la rentabilidad y el riesgo de sus inversiones. 
Tabla 1. Pros y contras del pago de dividendos

Table 1. Advantages and disadvantages of dividend payment

\begin{tabular}{|c|c|}
\hline Pros & Contras \\
\hline $\begin{array}{l}\text { 1. Los dividendos pueden ser atractivos para los } \\
\text { inversionistas que deseen flujos de efectivo estables, } \\
\text { pero que no quieran incurrir en costos de } \\
\text { transacción por la venta periódica de acciones. }\end{array}$ & $\begin{array}{l}\text { 1. Los dividendos se gravan como un ingreso } \\
\text { ordinario. }\end{array}$ \\
\hline $\begin{array}{l}\text { 2. Las finanzas conductuales argumentan que los } \\
\text { inversionistas con autocontrol limitado pueden } \\
\text { satisfacer las necesidades actuales de consumo con } \\
\text { acciones de altos rendimientos a la vez que se } \\
\text { adhieren a la política de nunca echar mano del } \\
\text { principal. }\end{array}$ & $\begin{array}{l}\text { 2. Los dividendos pueden reducir las fuentes internas } \\
\text { de financiamiento. Asimismo, pueden obligar a la } \\
\text { empresa a abandonar proyectos con VPN positivo o a } \\
\text { depender de un costoso financiamiento externo de } \\
\text { capital accionario. }\end{array}$ \\
\hline $\begin{array}{l}\text { 3. Los administradores, al proceder a favor de los } \\
\text { accionistas, pueden pagar dividendos con la finalidad } \\
\text { de alejar el efectivo de los tenedores de bonos. }\end{array}$ & $\begin{array}{l}\text { 3. Una vez que se haya establecido, las reducciones } \\
\text { de dividendos son difíciles de llevar a cabo sin afectar } \\
\text { negativamente el precio de las acciones de la } \\
\text { empresa. }\end{array}$ \\
\hline
\end{tabular}

4. El consejo de administración, al operar en

representación de los accionistas, puede usar los dividendos para reducir el efectivo disponible para el despilfarro de los administradores.

5. Los administradores pueden incrementar los dividendos para emitir una señal de su optimismo con respecto al flujo de efectivo futuro.

Fuente: (Ross et al., 2012, p. 600).

\section{La irrelevancia de la política de dividendos}

Para Miller y Modigliani (1961) la irrelevancia radica en que el pago de dividendos no tiene efectos sobre la riqueza de los accionistas, dado que un aumento del pago de los dividendos reduce en el mismo valor el precio de las acciones; las condiciones que hacen que la política de dividendos sea neutra están descritas en la Tabla 2. De esta forma el valor de la firma no depende de la política de dividendos, sino de la política de inversiones.

Tabla 2. Hipótesis básicas de la política de dividendos irrelevante

Table 2. Basic hypotheses of the dividend irrelevance theory

\begin{tabular}{cl}
\hline N.o & Hipótesis \\
\hline 1 & Los costos de transacción se ignoran. \\
\hline 2 & La política de inversión es constante. \\
\hline 3 & Las ganancias de capital y dividendos tienen el mismo gravamen. \\
\hline 4 & Los dividendos no transmiten información del mercado. \\
\hline 5 & Los mercados de valores son eficientes. \\
\hline 6 & Los inversionistas actúan de forma racional. \\
\hline & Fuente: elaboración propia, basado en Mascareñas (2011).
\end{tabular}


La lógica de esta irrelevancia, bajo el supuesto de mercados perfectos, es que un aumento en el pago de dividendos es seguido por el reemplazo de este dinero mediante la emisión de nuevas acciones para mantener el nivel de inversiones de la empresa (Booth \& Zhou, 2017), presentándose una transferencia de riqueza de los accionistas antiguos a los nuevos, equivalente al valor del pago de los dividendos; en consecuencia, el valor de cada acción disminuye en el mismo monto del dividendo pagado por el aumento en la cantidad de éstas; suponiendo que se conservan las hipótesis de la Tabla 2 .

\section{Teoría residual de los dividendos}

En esta teoría el pago de dividendos puede darse luego de evaluar y cubrir las necesidades de inversión; sólo cuando los beneficios obtenidos por la empresa son mayores a estas necesidades hay lugar al pago de dividendos (Pieloch-Babiarz, 2020), en caso contrario, no. Bajo esta teoría, la prioridad de los administradores es tener una estructura de capital óptima; por lo tanto, «el rendimiento requerido de los inversionistas, no se ve influenciado por la política de dividendos de la empresa, una premisa que a su vez implica que la política de dividendos es irrelevante» (Gitman \& Zutter, 2016, p. 579).

\section{Efecto clientela}

En el mercado de capitales los inversionistas no son homogéneos ni tampoco sus preferencias (Flavin \& O'Connor, 2017), esto conlleva al efecto clientela, el cual es definido por Ross et al., (2010) como «el hecho observable de que las acciones atraen a grupos específicos con base en los efectos fiscales y el rendimiento de los dividendos» (p.557); para complementar la definición, Castañeda et al., (2014) afirman al respecto que «inversionistas con altas tasas de impuestos a los dividendos demandarán acciones que pagan bajos dividendos y viceversa» (p.109).

Elton y Gruber (1970) a partir del estudio de dos variables: el rendimiento de las acciones y la relación de pago, entendida ésta como el pago de dividendos sobre las utilidades, identifican una racionalidad en el mercado en la que los accionistas en los tramos impositivos superiores muestran una preferencia por las ganancias de capital sobre los ingresos por dividendos en relación con aquellos en los grupos que pagan impuestos más bajos. En consecuencia, las tasas impositivas marginales que enfrenta el accionista determinan su comportamiento.

La hipótesis del efecto clientela, expuesto por Bajaj y Vijh (1990), implica que inversores con preferencias por acciones con dividendos altos presentan una mayor reacción frente a variaciones inesperadas del pago de dividendos cuanto mayor éstos sean, de ahí que los autores sostengan que hay una correlación perfecta entre el rendimiento de los dividendos y los anuncios inesperados del pago de estos. Según Gitman y Zutter (2016), como consecuencia del efecto clientela, una modificación en la política de dividendos no cambia el valor de la empresa, sino la base de propiedad de esta.

\section{La relevancia de la política de dividendos}

Cuando el pago de dividendos afecta el valor de las acciones en el mercado, y por consiguiente también el de la empresa, la política de dividendos es relevante; el argumento de Lintner (1956) y 
Gordon (1959), conocido como «más vale pájaro en mano», plantea que dada la incertidumbre que enfrentan los accionistas, éstos prefieren los dividendos actuales sobre los futuros, y como el precio de una acción equivale al valor actual de los dividendos futuros, entonces:

Los pagos de dividendos actuales reducen la incertidumbre de los accionistas y provocan que éstos descuenten las ganancias de la compañía a una tasa más baja y, en igualdad de condiciones, den un valor mayor a las acciones de la empresa. Por el contrario, si los dividendos se reducen o no se pagan, la incertidumbre de los accionistas aumentará, elevando el requerimiento y reduciendo el valor de las acciones. (Gitman \& Zutter, 2016, p.581).

Los mercados de capitales no son perfectos y enfrentan imperfecciones como los costos de transacción, costos de agencia, asimetrías de información y valor informativo de los dividendos (Makaew \& Maksimovic, 2020). En estos casos, la política de dividendos es relevante.

\section{Costos de transacción}

Dada la imperfección de los mercados, para el inversionista y la empresa los costos de transacción en el mercado son importantes y modifican su comportamiento al momento de comprar y vender las acciones, no solo en el hecho del intercambio, sino también al momento de tener utilidades gravables; análogamente para la empresa (Govindaraj et al., 2020); Higgins (1972), por medio de un modelo de optimización de dividendos demuestra que se puede maximizar la riqueza de los accionistas minimizando los costos de transacción, garantizando por una parte la inexistencia de excesos de liquidez en el caso de una entrega insuficiente de dividendos y evitando la necesidad de capital externo por dividendos excesivos.

También Rozeff (1982) presenta un modelo de pago óptimo de dividendos en el cual el incremento de los dividendos reduce los costos de agencia, pero aumenta el costo de transacción del financiamiento externo. En este modelo el pago óptimo de dividendos minimiza la suma de los dos costos.

\section{Costos de agencia}

Para Easterbrook (1984) los dividendos pueden ser útiles para reducir los costos de gestión de la agencia y mantienen a las empresas en el mercado de capitales; allí el monitoreo sobre los gerentes tiene menor costo y puede ser útil para ajustar el nivel de riesgo asumido por los gerentes y las diferentes clases de inversionistas. Jensen (1986) plantea que los dividendos son un mecanismo mediador entre los intereses de los administradores que se enfocan en hacer crecer la firma, como acción que habla bien de su gestión y los intereses de los inversionistas que reciben parte de las utilidades por medio de los dividendos; así los administradores no «abusan» de su poder en el manejo de los recursos.

\section{Asimetrías de información}

Otra imperfección del mercado es que la información no es completa sino asimétrica; es decir, que la información disponible en el mercado no es la misma para compradores y vendedores, como señala Akerlof (1970), al plantear que los vendedores de los bienes poseen más información que los 
compradores; por lo tanto, se generan incentivos para enviar señales falsas al mercado por parte de los vendedores, lo que puede terminar en un problema de "selección adversa» que afecta al mercado.

Bhattacharya (1979) plantea un modelo de dos periodos con información asimétrica en el cual los outsiders tienen información incompleta y el pago de dividendos se constituye en una señal de los flujos de efectivo esperados por la firma, de esta forma los directivos envían señales al mercado sobre la calidad de sus proyectos de acuerdo con el valor del dividendo. La asimetría de la información supone que ésta es escasa y por lo tanto no tenerla es un costo, Miller y Rock (como se citó en Sánchez Gálvez, 2012) quienes afirman que:

Los insiders, al manejar mejor y más información sobre las verdaderas ganancias y pérdidas de la empresa, enviarán señales al mercado por medio del pago de dividendos que escojan. Estas señales tienen relación con ganancias presentes y futuras (o esperadas), y que no son observables por los outsiders (p. 10).

\section{Valor informativo de los dividendos}

Presentar al mercado noticias buenas sobre las firmas para elevar el valor de éstas es un asunto tratado por Ross (1977). Su modelo muestra que el nivel de deuda y el pago de dividendos están interrelacionados en la determinación de una estructura óptima de capital y que los accionistas responden a las señales enviadas por los gerentes en desarrollo de sus funciones, suponiendo perfección y competencia en los mercados financieros.

Otro modelo de señalización con dividendos es tratado por John y Williams (1985). Los resultados llevaron a concluir que los dividendos más grandes (más pequeños) están asociados, ceteris paribus, con precios de acciones más altos (más bajos); en este modelo los dividendos son gravados con impuestos y, por ende, los inversionistas comparan este costo de transacción con la valoración de las acciones para decidir su comportamiento de acuerdo con la información recibida.

Kumar (1988) parte del suavizamiento del pago de dividendos al suponer que los directivos tienen mayor aversión al riesgo que los inversionistas; una de las conclusiones del estudio es que los aumentos de dividendos (disminuciones) señalan mejoras (deterioro) en las perspectivas de la empresa, pero aclara que los dividendos son malos predictores de las ganancias.

\section{Importancia de la política de dividendos en la empresa bajo el enfoque de la relevancia}

Sobre una muestra de 562 directores financieros de empresas que cotizan en la Bolsa de Valores de Nueva York, es pionero el estudio de Baker et al., (1985); hallan resultados a favor de la teoría de la señalización en el pago de dividendos, como consecuencia de las respuestas en cuanto a que los dividendos son útiles para analizar las perspectivas futuras de la compañía, el mercado por medio de anuncios de dividendos evalúa el valor de las acciones y que la empresa debe revelar adecuadamente los motivos de los cambios en la política de dividendos.

Baker y Powell (1999) investigan la opinión de 603 directores financieros de empresas que cotizan en la Bolsa de Valores de Nueva York sobre la relación entre política de dividendos y valor, mediante una 
encuesta aplicada en tres industrias diferentes (manufactura, comercio y servicios públicos); encuentran que para la mayoría de los directores la política de dividendos es relevante, asociada con la explicación del valor informativo (señalización) y se preocupan por la continuidad en el pago de dividendos. Cabe resaltar que las opiniones cambiaron poco entre industrias.

La Porta et al., (2000), en el estudio sobre políticas de dividendos en 4000 compañías de 33 países de todo el mundo, encuentran que las políticas de dividendos de las empresas pueden transmitir información a algunos inversores, la principal conclusión es:

Las empresas que operan en países con una mejor protección de los accionistas minoritarios pagan dividendos más altos. Además, en estos países, las empresas de rápido crecimiento pagan dividendos más bajos que las empresas de crecimiento lento, de acuerdo con la idea de que los accionistas legalmente protegidos están dispuestos a esperar sus dividendos cuando las oportunidades de inversión son buenas. Por otro lado, los accionistas poco protegidos parecen tomar los dividendos que pueden obtener, independientemente de las oportunidades de inversión (p. 27).

Smith (2011), luego de analizar resultados de encuestas aplicadas a directores financieros corporativos, concluye que las actitudes de los gerentes respaldan la hipótesis de la relevancia del dividendo. Chahyadi y Salas (2012) midieron los cambios en la propensión a pagar dividendos entre 1978 y 1998 en Estados Unidos, los resultados sugieren que las empresas tienen una propensión levemente menor a pagar dividendos respecto a los años anteriores; otros estudios relacionados con este fenómeno lo explican como resultado de una operación sustituta, la recompra de acciones por parte de las empresas.

Deshmukh et al., (2013) plantean un modelo que establece que el nivel de pago de dividendos es más bajo en las empresas administradas por CEO con exceso de confianza y que esta reducción es menor en las empresas de mayor crecimiento. Recientemente, Baker y Weigand (2015) presentaron una revisión de trabajos enfocados al estudio de la política de dividendos; uno de los resultados apunta a la hipótesis de la relevancia, «las empresas tienden a seguir una política de dividendos suavizados en lugar de una política de dividendos residuales» (p.126).

A manera de conclusión a favor de la relevancia, "en la actualidad la mayoría de los gerentes financieros, especialmente los que manejan grandes corporaciones, creen que la política de pagos puede afectar el valor de la empresa» (Gitman \& Zutter, 2016, p.581), y en cuanto a su complejidad «Cuanto más nos fijamos en la imagen del dividendo, más parece un rompecabezas, con piezas que simplemente no encajan» (Black, 1976, p.5).

\section{METODOLOGÍA}

En este texto de revisión literaria, se abordaron los principales autores y sus aportes en el desarrollo teórico de la política de dividendos, incluyendo las dos ramas fundamentales: relevancia e irrelevancia; luego, se procedió a buscar trabajos empíricos que dieran cuenta de la aplicación del cuerpo teórico en la toma de decisiones financieras en las empresas; sin embargo, dada la heterogeneidad de las organizaciones en el ámbito mundial, se restringió la búsqueda a Iberoamérica, donde la concentración de la propiedad en las organizaciones es mayor y la regulación 
es laxa en contraste con Europa y Estados Unidos; se incluyeron algunas investigaciones realizadas en España debido a la riqueza en datos y aplicación estadística.

Se utilizaron las bases de datos Scopus, Ebsco, Redalyc y Google académico, los criterios para la búsqueda fueron combinaciones de las palabras clave: dividendo societario, política de dividendos, pago de dividendos; en la selección de documentos se tuvo en cuenta que en el título aparezca el nombre de países iberoamericanos, alusiones directas a las clases de políticas de dividendos, metodologías cuantitativas y estudios empíricos; en cuanto al idioma se seleccionaron documentos en español y portugués. Para aclarar conceptos se acudió a libros de texto de las principales editoriales como Pearson, McGraw-Hill y John Wiley \& Sons.

Las investigaciones revisadas que cumplieron la totalidad de los requisitos fueron 29, las cuales se clasificaron en tres categorías de acuerdo con el enfoque de la política de dividendos plasmada. En el primer grupo, las investigaciones bajo el enfoque de la irrelevancia, en el segundo las del enfoque de la relevancia y finalmente las investigaciones que no siguieron algunos de los enfoques, que corresponden al $14 \%, 72 \%$ y $14 \%$ respectivamente de la muestra total. Al comparar entre países, la mayoría de la literatura corresponde a estudios realizados en Brasil, con el $28 \%$ de los documentos.

Los artículos de investigación sobre el tema son escasos. Al realizar la búsqueda inicial en Scopus, los mayores resultados se logran con la combinación «política» y «dividendos», en cuyo caso arroja trece (13) documentos en los últimos veinticinco (25) años. Sin embargo, la mayoría no cumplen los criterios expuestos con anterioridad, por lo que fue necesario acudir a las otras bases de datos. Las variables objeto de análisis en los documentos seleccionados son: distribución por países, metodología aplicada, enfoques de la política de dividendos, teorías alrededor de dichos enfoques y resultados.

\section{RESULTADOS}

En torno a la discusión sobre la relevancia e irrelevancia se empezaron a desarrollar trabajos empíricos para identificar los determinantes de la política de dividendos en Iberoamérica, principalmente en Brasil, Chile y España. Para el caso colombiano se encontraron resultados en tres investigaciones, como puede verse en la Tabla 3. Iberoamérica está conformada por 22 países; en el presente estudio se mencionan nueve, lo que corresponde al $41 \%$ del territorio.

En Colombia, las empresas están obligadas a entregar dividendos a sus accionistas, como mínimo, del $50 \%$ de sus utilidades; sin embargo, este porcentaje puede ser menor, e incluso cero, si más del $78 \%$ del valor de las acciones representadas así lo deciden, como lo indica el artículo 155 del Código de Comercio. A manera de comparación, se tiene que las empresas en Chile están obligadas a entregar, como mínimo, el 30\% de las utilidades a sus accionistas vía dividendos (artículo 79 de la Ley sobre Sociedades Anónimas); en Argentina, como mínimo, el 20\% (artículo 320 de la Ley de Sociedades Comerciales); y, en Brasil, como mínimo el 25\% (Ley de las Sociedades Anónimas). 
Tabla 3. Estudios en países Iberoamericanos Table 3. Studies in Ibero-America

\begin{tabular}{lc}
\hline Países & Cantidad \\
\hline Argentina & 1 \\
Brasil & 8 \\
Chile & 6 \\
Colombia & 2 \\
Colombia, México, Perú y Chile* & 1 \\
Ecuador & 1 \\
España & 4 \\
España, & 1 \\
México & 1 \\
Perú & 2 \\
Venezuela & 2 \\
\hline Total general & 1 \\
\hline
\end{tabular}

Nota: *Corresponde a investigaciones que tomaron en la muestra empresas de diferentes países que cotizan en las Bolsas de Valores Correspondientes.

Fuente: elaboración propia.

La metodología usada en las investigaciones se muestra en la Tabla 4, la mayoría corresponde a datos de panel, donde la variable explicada corresponde a una razón de pago de dividendos sobre los beneficios en la mayoría de los casos; le siguen los modelos binarios Logit, allí la variable que se explica es la decisión de pagar o no dividendos; aparte de éstos, se encuentran los modelos econométricos lineales, estocásticos y no paramétricos; sin embargo, las variables en los modelos en su gran mayoría son financieras y se agrupan alrededor de la inversión, activos, ventas, endeudamiento, patrimonio, rentabilidad y pago de dividendos; también se toman variables macroeconómicas como el Producto Interno Bruto (PIB) y la inflación e información del mercado como los precios históricos de las acciones.

Tabla 4. Metodología aplicada en los estudios

Table 4. Methodology applied in the studies

\begin{tabular}{ll}
\hline \multicolumn{1}{c}{ Metodología } & Cantidad \\
\hline Análisis cuantitativo de tasas de rentabilidad & 1 \\
\hline Cálculo de indicadores financieros de endeudamiento y reinversión & 1 \\
\hline Censo poblacional (encuesta) & 1 \\
\hline Datos de panel & 11 \\
\hline Estudio de eventos & 1 \\
\hline Modelo de correlación de Spearman & 1 \\
\hline Modelo de descuento de dividendos & 1 \\
\hline Modelo estocástico de tipo caminata aleatoria & 1 \\
\hline Modelo Logit & 6 \\
\hline Portafolios de alto y bajo rendimiento del dividendo & 1 \\
\hline Regresión lineal múltiple & 2 \\
\hline Regresiones lineales multivariadas por MCO & 1 \\
\hline Series de tiempo & 1 \\
\hline Total general & 29 \\
\hline
\end{tabular}

Fuente: elaboración propia. 
Frente a los enfoques de la política de dividendos, en la Tabla 5, se observan las teorías que se abordan en las investigaciones analizadas; el efecto clientela es el más estudiado, aparece en 3 de los 4 documento del enfoque de la irrelevancia; por otra parte, la teoría del valor informativo de los dividendos es la más contrastada bajo el enfoque de la relevancia, en forma independiente en doce casos y en conjunto con los costos de agencia en dos. En cuatro de los estudios analizados no se presentaron estos enfoques.

Tabla 5. Enfoques de la política de dividendos

Table 5. Approaches to dividend policy

\begin{tabular}{clc}
\hline Enfoque & \multicolumn{1}{c}{ Teoría aplicada } & Cantidad \\
\hline \multirow{2}{*}{ Irrelevancia } & Efecto clientela & 3 \\
& Teoría de dividendos residuales & 1 \\
\hline \multirow{2}{*}{ Relevancia } & Costos de agencia & 7 \\
& Valor informativo de los dividendos & 12 \\
& Valor informativo de los dividendos y Costos de agencia & 2 \\
\hline No aplica & No aplica & 4 \\
\hline Total general & & 29
\end{tabular}

En la Tabla 6, se presentan los autores e investigaciones que tratan los enfoques de la política de dividendos. El efecto clientela es abordado por Gea Sánchez (2016) mediante el uso de Coeficientes del modelo de correlación de Spearman (modelo no paramétrico) sobre una muestra de acciones de empresas que cotizan en la Bolsa de Comercio de Buenos Aires, los resultados no son concluyentes, finalmente se inclina por la teoría de la relevancia, pero el modelo no es lo suficientemente robusto. El tema también es estudiado por Gomes Novaes et al., (2015), pero los resultados de los modelos propuestos no permitieron aceptar la hipótesis del efecto clientela como explicación del pago de dividendos adicionales en Brasil.

Pinheiro y Dias (2012) no descartaron plenamente la hipótesis sobre la relación entre la declaración de dividendos y el efecto clientela al comparar los resultados que obtuvieron en sus operaciones empresas en Brasil que entregaron dividendos y los que no lo hicieron, teniendo en cuenta la existencia de accionistas controladores, porcentaje de propiedad del accionista mayor, bursatilidad de las acciones y la cantidad de accionistas, entre otros. Vancin y Laser Procianoy (2016) al profundizar en la teoría de dividendos residuales encontraron que el 63.3\% de las empresas en Brasil pagan dividendos por encima del mínimo; recalcan que la verdadera política de dividendos aplica cuando se paga por encima del valor mínimo; las empresas solo pagan dividendos si presentan acumulación de utilidades, que es lo que caracteriza esta teoría. 
Tabla 6. Investigaciones seleccionadas que tratan teorías de la irrelevancia

Table 6. Selected studies that address dividend irrelevance theories

\begin{tabular}{|c|c|c|}
\hline Teoría tratada & Autores & Título de la investigación \\
\hline \multirow{3}{*}{ Efecto clientela } & Gea Sánchez (2016) & $\begin{array}{l}\text { Comprobaciones empíricas de las teorías sobre políticas de } \\
\text { dividendos en el Mercado de Valores de Buenos Aires durante el } \\
\text { período 2006-2011 }\end{array}$ \\
\hline & $\begin{array}{l}\text { Gomes Novaes, et al., } \\
(2015)\end{array}$ & $\begin{array}{l}\text { Efeito clientela: uma análise da relação entre book-tax diferencias e } \\
\text { dividendos extraordinários das empresas com ações listadas na } \\
\text { Bovespa }\end{array}$ \\
\hline & Pinheiro y Dias (2012) & Dividendos e efeito clientela: Evidencias no mercado brasileiro \\
\hline $\begin{array}{l}\text { Teoría de } \\
\text { dividendos } \\
\text { residuales }\end{array}$ & $\begin{array}{l}\text { Vancin y Laser Procianoy } \\
\text { (2016) }\end{array}$ & Índices contábeis e a decisão do pagamento de dividendos \\
\hline
\end{tabular}

Las investigaciones relacionadas con el enfoque de la relevancia se dividen en tres grupos: Costos de agencia, Valor informativo de los dividendos y un tercer grupo que es una combinación de los anteriores. En la Tabla 7, se presentan 21 estudios, en su gran mayoría de la teoría del Valor informativo de los dividendos.

Tabla 7. Investigaciones seleccionadas que tratan teorías de la relevancia

Table 7. Selected studies that address dividend relevance theories

\begin{tabular}{|c|c|c|}
\hline Teoría tratada & Autores & Título de la investigación \\
\hline \multirow{7}{*}{$\begin{array}{l}\text { Costos de } \\
\text { agencia }\end{array}$} & Lozano et al., (2002) & Papel de la política de dividendos en las empresas reguladas. \\
\hline & $\begin{array}{l}\text { Gutiérrez Urzúa et al., } \\
\text { (2012) }\end{array}$ & Análisis de la política de pago de dividendos en empresas chilenas. \\
\hline & Lefort (2008) & $\begin{array}{l}\text { El efecto de los conflictos de agencia en las políticas de dividendos a } \\
\text { los accionistas. El caso chileno. }\end{array}$ \\
\hline & $\begin{array}{l}\text { López Iturriaga y } \\
\text { Rodríguez Sanz (1999) }\end{array}$ & $\begin{array}{l}\text { La decisión de dividendos en la empresa española: un contraste de } \\
\text { teorías alternativas. }\end{array}$ \\
\hline & $\begin{array}{l}\text { López Iturriaga y Saona } \\
\text { Hoffmann (2007) }\end{array}$ & $\begin{array}{l}\text { Endeudamiento, dividendos y estructura de propiedad como } \\
\text { determinantes de los problemas de agencia en la gran empresa } \\
\text { española. }\end{array}$ \\
\hline & $\begin{array}{l}\text { Maquieira V. y Moncayo } \\
\text { M. (2004) }\end{array}$ & $\begin{array}{l}\text { Costos de Agencia y Costos de Transacción como determinantes de } \\
\text { la tasa de pago de dividendos en Chile: Extensión. }\end{array}$ \\
\hline & San Martín Reyna (2017) & $\begin{array}{l}\text { Estructura de propiedad y su efecto en la política de dividendos en } \\
\text { el contexto mexicano. }\end{array}$ \\
\hline $\begin{array}{l}\text { Valor } \\
\text { informativo de } \\
\text { los dividendos }\end{array}$ & Álvarez Agudelo (2013) & $\begin{array}{l}\text { ¿Qué impacto tiene sobre la rentabilidad de un inversionista la } \\
\text { política de distribución de utilidades? Una mirada desde el } \\
\text { accionista colombiano. }\end{array}$ \\
\hline
\end{tabular}


Cabrera Tobar (2019) La política de dividendos y su incidencia en la rentabilidad de las empresas grandes del sector de calzado del Ecuador.

\begin{tabular}{|c|c|}
\hline $\begin{array}{l}\text { De Melo y Wagner da } \\
\text { Fonseca (2015) }\end{array}$ & $\begin{array}{l}\text { Politicas de dividendos no Brasil: uma análise na reacao do mercado } \\
\text { a anúncios de distribuicao de proventos. }\end{array}$ \\
\hline Galvão et al., (2018) & $\begin{array}{l}\text { Dividendos, juros sobre capital próprio e níveis de payout : Um } \\
\text { estudo investigativo sobre a política de distribuição de dividendos } \\
\text { adotada pelas empresas listadas na BM \& Fbovespa }\end{array}$ \\
\hline $\begin{array}{l}\text { Giner Bagüés y Salas } \\
\text { Fumás (1995) }\end{array}$ & $\begin{array}{l}\text { Explicaciones alternativas para la política de dividendos: análisis } \\
\text { empírico con datos empresariales españoles. }\end{array}$ \\
\hline $\begin{array}{l}\text { Kristjanpoller Rodríguez y } \\
\text { Silva Arroyo (2014) }\end{array}$ & $\begin{array}{l}\text { El impacto de la propiedad institucional de las administradoras de } \\
\text { fondos de pensión sobre la política de dividendos: evidencia para } \\
\text { Chile. }\end{array}$ \\
\hline Leite et al., (2018) & $\begin{array}{l}\text { Revisitando os determinantes da distribuição de dividendos: uma } \\
\text { análise em empresas brasileiras. }\end{array}$ \\
\hline $\begin{array}{l}\text { Maquieira V. y Osorio M. } \\
(2000)\end{array}$ & $\begin{array}{l}\text { Anuncios de cambios en pagos de dividendos y su impacto en la } \\
\text { riqueza de los accionistas: evidencia empírica en Chile. }\end{array}$ \\
\hline $\begin{array}{l}\text { Menezes da Silva et al., } \\
\text { (2016) }\end{array}$ & A relevância do dividendo adicional proposto. \\
\hline $\begin{array}{l}\text { Mongrut Montalvan et } \\
\text { al., (2017) }\end{array}$ & $\begin{array}{l}\text { Política de dividendos y buen gobierno corporativo en el Perú: } \\
\text { ¿Existe alguna relación? }\end{array}$ \\
\hline $\begin{array}{l}\text { Rodrigues Sobrinho et al., } \\
\text { (2014) }\end{array}$ & $\begin{array}{l}\text { Dividendos y acumulaciones discricionários: um estudo sobre una } \\
\text { relación entre una política de distribución de dividendos ea } \\
\text { qualidade dos lucros. }\end{array}$ \\
\hline Sánchez Gálvez (2012) & $\begin{array}{l}\text { Determinantes de la Política de Dividendos en Chile: años 2007- } \\
2010\end{array}$ \\
\hline $\begin{array}{l}\text { García-Borbolla } \\
\text { Fernández (2003) }\end{array}$ & $\begin{array}{l}\text { La relevancia de los dividendos para explicar el valor de mercado de } \\
\text { las acciones. }\end{array}$ \\
\hline Núñez Pérez et al., (2013) & Modelo de política óptima de dividendos. \\
\hline
\end{tabular}
Fuente: elaboración propia.

Los estudios que analizan la problemática de los costos de agencia se concentran en España, Chile y México; en el mercado bursátil español, Lozano et al., (2002) verifica que las empresas reguladas utilizan la política de dividendos para mitigar los conflictos de agencia y muestran que están más incentivadas a acudir a los mercados de capitales utilizando la política de dividendos. La muestra está constituida por las empresas no financieras registradas en la Comisión Nacional del Mercado de Valores de España, la regulación tiene que ver con la fijación de precios máximos en las industrias y por regímenes especiales de impuestos. En este estudio se concluye que frente a un límite establecido en los precios los accionistas reciben sus beneficios vía dividendos. López Ituriaga y Rodríguez Sanz (1999) se proponen verificar la teoría de las clientelas fiscales, pero no encuentran soporte en la investigación; corroboran parcialmente las hipótesis derivadas de la teoría de agencia, 
al encontrar correlación positiva con el tamaño de la empresa y negativa con el endeudamiento y concentración de la propiedad. López Iturriaga y Saona Hoffmann (2007) concluyen que el pago de dividendos desempeña una «función disciplinante» sobre los directivos de las compañías en España.

En lo referente a resultados de las investigaciones en Chile, Maquieira V. y Moncayo M. (2004) encuentran que los administradores usan los dividendos como un mecanismo de transmisión de información al mercado de sus expectativas de flujos presentes y futuros. Lefort (2008) indica que en las empresas chilenas con mayor nivel de conflictos de intereses tienden a pagar un dividendo más alto a los inversionistas, como en el caso de las empresas que pertenecen a grupos económicos. Finalmente, Gutiérrez Urzúa et al., (2012) en su estudio evidencian de la utilización de la tasa de pago de dividendos para entregar información sobre los proyectos futuros de la empresa y el tratamiento justo de los accionistas.

Para San Martín Reyna (2017) la concentración de la propiedad en la empresa familiar en México tiene un efecto negativo en la distribución de dividendos, quien aborda el estudio por la teoría de la agencia; sin embargo, con el ingreso de inversionistas el efecto se revierte, por lo que la democratización de las empresas se considera una estrategia de beneficios basada en el pago de dividendos para los nuevos copropietarios.

La teoría del valor informativo de los dividendos se abordó principalmente en Brasil. Las investigaciones donde no se pudo comprobar esta teoría fueros las realizadas por Rodrigues Sobrinho et al., (2014), cuyo estudio buscó relacionar la política de dividendos con la calidad de las ganancias. Sin embargo, los resultados no permitieron establecer una relación clara entre el pago de dividendos y las ganancias; De Melo y Wagner da Fonseca (2015). En este estudio se descarta que hacer anuncios sobre el pago de dividendos tiene incidencia en el mercado.

Los estudios donde la evidencia estuvo a favor de la teoría del valor informativo de los dividendos en Brasil son los de Menezes da Silva et al., (2016). Los investigadores hallaron evidencias en forma de la hipótesis de relevancia mediante la divulgación del Dividendo adicional propuesto (DAP); Galvão et al., (2018) encontraron que en un 60\% las empresas pagan dividendos por encima del mínimo, que corresponde al $25 \%$ de las utilidades, encuentran una tendencia a pagar dividendos en todos los años. En Leite et al., (2018) los resultados demostraron que la rentabilidad, el tamaño de la empresa y la distribución de dividendos en el año anterior son los factores que contribuyen de forma positiva y significativa en el pago de dividendos, y contradicen a Galvão et al., (2018), indicando que el pago de dividendos no es constante, sino que depende del desempeño financiero y las expectativas de los inversionistas.

Para el caso Chileno, Maquieira V. y Osorio M. (2000) concluyeron que los dividendos transmiten información de la situación financiera de la empresa; por su parte Sánchez Gálvez (2012), usando modelos de regresión lineal, no llega a resultados confiables a favor de la teoría de la señalización en Chile, argumentando que se puede deber al tamaño de la muestra lo que no permite niveles de significancia suficientes; Kristjanpoller Rodríguez y Silva Arroyo (2014) obtienen una relación positiva y significativa entre el nivel de propiedad de las Administradoras de Fondos de Pensiones (AFP) chilenas y el pago de dividendos de las empresas en las cuales invierten. 
Álvarez Agudelo (2013) concluye que las empresas que reparten mayor dividendo en Colombia han presentado un crecimiento constante en sus utilidades netas; Mongrut Montalvan et al., (2017) encuentran una relación positiva entre el pago de dividendos y el establecimiento de un gobierno corporativo, lo cual se constituye como una señal para los inversionistas en Perú. Cabrera Tobar (2019) concluye que las empresas en Ecuador pagan dividendos inclusive en los periodos de pérdidas por medio de utilidades acumuladas; la política de dividendos está relacionada directamente con el control de utilidades, estas empresas no cotizan en bolsa.

Giner Bagüés y Salas Fumás (1995) encuentran que la política de dividendos de la empresa "es relativamente rígida de acuerdo a las pautas del modelo propuesto por Lintner (1956), pero la hipótesis de que esa política es insensible a cambios en las condiciones económicas en que se desenvuelven las empresas se rechaza» (p.346). La explicación planteada es que teniendo en la muestra dos tipos de empresas, unas con alta y otras con baja participación bancaria, observaron que existe una sensibilidad frente a los efectos de la señalización del mercado y las asimetrías de la información respectivamente. En cuanto al desarrollo teórico alrededor de los dividendos, Fernández (1987) proporciona una visión global del asunto, concluye que en la práctica los gerentes para la toma de decisiones asignan un valor informativo de los dividendos.

Finalmente, los autores que analizan en forma conjunta el valor informativo de los dividendos y los costos de agencia se encuentran el final de la Tabla 8; García-Borbolla Fernández (2003) halla en países europeos evidencia a favor de la relevancia de los dividendos con la aplicación de la teoría de señales y costos de agencia; la muestra estuvo conformada por empresas no financieras de Alemania, España, Francia, Holanda, Italia, Suiza y Reino Unido, con un total de 16.185 observaciones. Núñez Pérez et al., (2013) establecen la posible validación de las lógicas del valor informativo de los dividendos y de costos de agencia en el mercado mexicano de valores.

Tabla 8. Investigaciones seleccionadas fuera de los enfoques de relevancia e irrelevancia Table 8. Selected studies outside relevance and irrelevance theories

\begin{tabular}{lll}
\hline Teoría tratada & \multicolumn{1}{c}{ Autores } & \multicolumn{1}{c}{ Título de la investigación } \\
\hline & $\begin{array}{l}\text { Betancur-Duque y } \\
\text { Jiménez-Echeverri (2019) }\end{array}$ & $\begin{array}{l}\text { Valoración de acciones en los países que integran la Alianza del } \\
\text { Pacífico por el modelo de descuento de dividendos. }\end{array}$ \\
\cline { 2 - 3 } No aplica & $\begin{array}{l}\text { Hernández-Chacín et al., } \\
(2017)\end{array}$ & $\begin{array}{l}\text { Decisiones sobre dividendos en hipermercados y supermercados } \\
\text { familiares en el estado Zulia, Venezuela. }\end{array}$ \\
\cline { 2 - 3 } & Santos (2008) & La política de dividendos y la rentabilidad de los negocios. \\
\cline { 2 - 3 } & $\begin{array}{l}\text { Usma Patiño y Ramírez } \\
\text { Arango (2010) }\end{array}$ & Gobierno corporativo y política de dividendos: el caso colombiano. \\
\hline
\end{tabular}

Fuente: elaboración propia.

Otras investigaciones sobre distribución de dividendos se muestran en la tabla 8, tienen en común que no se desarrollan alrededor de las políticas de dividendos. Santos Jiménez (2008) Analiza la política de dividendos de las empresas del sector de generación eléctrica que cotizan en la Bolsa de Valores de Lima; la muestra está representada por cuatro empresas, el estudio no llega a resultados concretos frente a la aplicación de la política de dividendos. 
Usma Patiño y Ramírez Arango (2010), por su parte, mostraron para el caso colombiano que mayores ratios de pago de dividendos han estado asociados a mejores índices de Gobierno Corporativo, con una muestra de 279 empresas y datos entre 1997 y 2008 inscritas en el Registro Nacional de Valores y Emisores, tomando empresas con y sin Gobierno Corporativo y que pagaron o no dividendos en los diferentes años del periodo establecido. Por medio de un modelo de panel de datos hallaron correlación entre el pago de dividendos y las variables estadísticamente significativas: tamaño de la empresa (positiva) y el apalancamiento (negativa).

En las empresas familiares la distribución de dividendos es más compleja que en la gran empresa, como lo indican Llorente Muñoz (2012) y Hernández-Chacín et al., (2017), por las relaciones que se dan a partir de la triada familia, empresa y propiedad, donde los factores afectivos y emocionales amenazan la eficiencia de la empresa, la solución planteada es la creación de un protocolo familiar. Este documento es el punto de partida en la solución de conflictos que nacen, principalmente, porque no hay una división entre la propiedad y la administración.

Betancur-Duque y Jiménez-Echeverri (2019) al estudiar las oportunidades de inversión en países pertenecientes a la Alianza del Pacífico plantean una discusión que apuntaría a la irrelevancia de la política de dividendos, al identificar que la mayoría de las acciones de la muestra están sobrevaloradas y en consecuencia no reflejan el valor real de la empresa. De las 132 acciones estudiadas el $87.88 \%$ presentan un precio de mercado por encima del valor intrínseco, en Colombia corresponde al $73.33 \%$, en México el $89.13 \%$, en Perú el $63.64 \%$ y en Chile el $90.16 \%$.

\section{DISCUSIÓN}

En este documento de revisión de literatura, acerca de la evolución teórica de las políticas de dividendos y al mismo tiempo de estudios empíricos encausados a identificar sus determinantes, se destaca que en economías en desarrollo ubicadas en Iberoamérica, donde los mercados de capitales son débiles en comparación con las potencias mundiales, se encuentran estudios con metodologías estadísticamente robustas que ayudan a entender la evolución de las organizaciones referente a toma de decisiones financieras y el desarrollo del mercado de capitales.

El valor agregado de esta investigación está en identificar cronológicamente le evolución de la política de dividendos, los aportes de sus autores y los esfuerzos de investigadores en trabajos empíricos por entender cómo se aplican en la realidad. La política de dividendos es relevante cuando el pago de dividendos está relacionado con el valor de la firma, en caso contrario la política de dividendos es estéril, en el sentido que trae las mismas consecuencias pagar o no dividendos de las utilidades de la organización sobre el valor de esta; siendo un tema tan sensible, todas las empresas deberían incorporarla entre sus objetivos financieros de corto plazo.

Los países donde más trabajos empíricos se presentan son Brasil, Chile y España. Del total de trabajos, el $72 \%$ corresponde al enfoque de la relevancia, más que todo a la teoría del valor informativo de los dividendos; aunque las metodologías cuantitativas utilizadas gozan de reconocimiento científico, en algunos casos los resultados no fueron concluyentes por los tamaños muestrales insuficientes, dado que en la región las Bolsas de Valores aún están en desarrollo a excepción de España. Sin embargo, la cantidad de trabajos encontrados es reducida. Para próximos ejercicios se sugiere relajar los 
criterios de selección e incluir estudios cualitativos que den cuenta de otras variables aparte de las financieras para entender el problema de la distribución de dividendos en la región.

Al consultar las bases de datos especializadas, se observa que las publicaciones relacionadas con política de dividendos han venido disminuyendo, puede ser por las dinámicas mundiales de la economía en la actualidad, donde el interés por la democratización de la propiedad es menor y en consecuencia pierde vigencia el problema de los dividendos con la concentración de la propiedad, como se ha expuesto en investigaciones citadas en este documento; sin embargo, tomando en cuenta las expectativas de crecimiento del mercado de capitales en la región y el interés de las nuevas generaciones por activos financieros como las monedas virtuales, mercado de divisas y acciones, sería de gran interés plantear estudios comparativos de sus beneficios; por otra parte, de acuerdo a la evidencia empírica, las empresas tienen un incentivo con el pago de dividendos, porque esto puede mejorar el valor de la firma en el mercado; no obstante, es preciso establecer en qué medida podría ocurrir si las acciones están sobrevaloradas en el mercado.

\section{CONCLUSIONES}

En la región se observa que recientemente han disminuido los estudios cuantitativos sobre política de dividendos, a excepción de Brasil, donde se evidencian investigaciones sobre ambos enfoques de esta política, en segundo lugar, está Chile: entre ambos se acumula el $48 \%$ de las investigaciones analizadas; esto podría guardar relación directa con los porcentajes mínimos obligados de entrega de utilidades vía dividendos a sus accionistas, que en Brasil es del $25 \%$ y en Chile del $30 \%$. Entre mayor sea este porcentaje mínimo, mayor será el interés por analizar la política de dividendos.

Entre las metodologías cuantitativas para estudiar los determinantes de la política de dividendos, son privilegiados los datos de panel, gracias a que toman simultáneamente datos longitudinales y transversales de las organizaciones, es decir, datos de distintas organizaciones en distintos momentos de tiempo para elaborar modelos predictivos. Adicionalmente, proveen mayor heterogeneidad, eficiencia y consistencia estadística.

La evidencia empírica se desarrolla alrededor del estudio de las variables relacionadas con el pago de los dividendos, en su mayoría como una razón de pago de dividendos respecto a los beneficios de la firma, los resultados van enfocados a identificar dichas variables y determinar si la política de dividendos es o no relevante. La mayor parte de los estudios están asociados a la relevancia de la política de dividendos y la comprobación de sus hipótesis los llevó a aceptar las teorías de costos de agencia y valor informativo de los dividendos para explicar la política de dividendos en las compañías representativas de sus países.

\section{CONFLICTOS DE INTERÉS}

Los autores declaran que no presentan conflictos de interés financiero, profesional o personal que pueda influir de forma inapropiada en los resultados obtenidos o las interpretaciones propuestas. 


\section{CONTRIBUCIÓN DE AUTORES}

Para el desarrollo de este proyecto todos los autores han realizado una contribución significativa especificada a continuación:

Albeiro Aguirre-Ríos: estructuración de artículo, revisión y desarrollo conceptual y teórico.

Jovany Sepúlveda-Aguirre: revisión y estructuración de forma del artículo, revisión de citas y referencias, apoyo en la redacción y cohesión del texto.

Uvenny Quirama-Estrada: apoyo en la redacción de resultados y discusión, revisión del texto.

Luis Fernando Garcés Giraldo: revisión y estructuración de forma del artículo, revisión de citas y referencias, apoyo en la redacción y cohesión del texto, revisión y ajuste de versiones del texto.

\section{REFERENCIAS}

Akerlof, G. A. (1970). The market for «Lemons»: Quality uncertainty and the market mechanism. The Quartely Journal of Economics, v. 84, n. 3, 488-500. https://doi.org/10.2307/1879431

Álvarez Agudelo, A. (2013). ¿Qué impacto tiene sobre la rentabilidad de un inversionista la política de distribución de utilidades?: una mirada desde el accionista colombiano (Tesis de maestría). URL

Bajaj, M.; Vijh, A. M. (1990). Dividend clienteles and the information content of dividend changes. Journal of Financial Economics, v. 26, n. 2, 193-219.

https://doi.org/10.1016/0304-405X(90)90003-1

Baker, H. K.; Powell, G. E. (1999). How corporate managers view dividend policy. Quarterly Journal of Business and Economics, v. 38, n. 2, 17-27. URL

Baker, H. K.; Farrelly, G. E.; Edelman, R. B. (1985). A survey of management views on dividend policy. Financial Management, v. 14, n. 3, 78-84. URL

Baker, H. K.; Weigand, R. (2015). Corporate dividend policy revisited. Managerial Finance, v. 41, n. 2, 126-144. https://doi.org/10.1108/MF-03-2014-0077

Berk, J.; DeMarzo, P. (2010). Fundamentos de finanzas corporativas. Pearson.

Betancur-Duque, F. A.; Jiménez-Echeverri, E. A. (2019). Valoración de acciones en los países que integran la Alianza del Pacífico por el modelo de descuento de dividendos. Revista CEA, v. 5, n. 10, 99-116. https://doi.org/10.22430/24223182.1450

Bhattacharya, S. (1979). Imperfect information, dividend policy, and "the bird in the hand» fallacy. The Bell Journal of Economics, v. 10, n. 1, 259-270. https://doi.org/10.2307/3003330 
Black, F. (1976). The dividend puzzle. Journal of Portfolio Management, v. 2, n. 2, 5-8. https://doi.org/10.3905/jpm.1976.408558

Booth, L.; Zhou, J. (2017). Dividend policy: A selective review of results from around the world. Global Finance Journal, v. 34, 1-15. https://doi.org/10.1016/j.gfj.2017.07.002

Cabrera Tobar, V. N. (2019) La política de dividendos y su incidencia en la rentabilidad de las empresas grandes del sector de calzado del Ecuador (Tesis de maestría). URL

Castañeda, F.; Aguirre, J. P.; Ormazábal, F.; Contreras, F.; Madariaga, L. (2014). Manual de finanzas corporativas (2da ed.). Universidad de Santiago de Chile.

Chahyadi, C. S.; Salas, J. M. (2012). Not paying dividends? A decomposition of the decline in dividend payers. Journal of Economics and Finance, v. 36, n. 2, 443-462. https://doi.org/10.1007/s12197-010-9132-0

Davaadorj, Z. (2019). Does social capital affect dividend policy? Journal of Behavioral and Experimental Finance, v. 22, 116-128. https://doi.org/10.1016/j.jbef.2019.02.010

De Melo, F. J.; Wagner da Fonseca, M. (2015). Política de dividendos no Brasil: uma análise na reação do mercado a anúncios de distribuição de proventos. Revista Contemporânea de Contabilidade, v. 12, n. 27, 137-164. https://doi.org/10.5007/2175-8069.2015v12n27p137

Deshmukh, S.; Goel, A. M.; Howe, K. M (2013). CEO Overconfidence and divident policy. Journal of Financial Intermediation, v. 22, n. 3, 440-463. https://doi.org/10.2139/ssrn.1107542

Easterbrook, F. H. (1984). Two agency-cost explanations of dividends. The American Economic Review, v. 74, n. 4, 650-659. URL

Elton, E. J.; Gruber, M. J. (1970). Marginal stockholder tax rates and the clientele effect. The Review of Economics and Statistics, v. 52, n. 1, 68-74. https://doi.org/10.2307/1927599

Fernández, J. M. (1987) Discusión teórica y evidencia empírica acerca de la política de dividendos. Anales de Estudios Económicos y Empresariales, n. 2, 9-34. URL

Flavin, T.; O'Connor, T. (2017). Reputation building and the lifecycle model of dividends. Pacific-Basin Finance Journal, v. 46, part a, 177-190. $\underline{\text { URL }}$

Galvão, K. S.; dos Santos, J. F.; Araujo, J. M. (2018). Dividendos, juros sobre capital próprio e níveis de payout: Um estudo investigativo sobre a política de distribuição de dividendos adotada pelas empresas listadas na BM\&FBovespa. Revista Contemporânea de Contabilidade. v. 15, n. 36, 330. https://doi.org/10.5007/2175-8069.2018v15n36p3

García-Borbolla Fernández, A. (2003). La relevancia de los dividendos para explicar el valor de mercado de las acciones (Tesis de doctorado). $\underline{\mathrm{URL}}$ 
Gea Sánchez, L. J. (2016) Comprobaciones empíricas de las teorías sobre políticas de dividendos en el mercado de valores de Buenos Aires durante el período 2006-2011 (Tesis de maestría). URL

Giner Bagüés, E.; Salas Fumás, V. (1995). Explicaciones alternativas para la política de dividendos: Análisis empírico con datos empresariales españoles. Investigaciones económicas, v. 19, n. 3, 329-348. URL

Gitman, L. J.; Zutter, C. J. (2016). Principios de administración financiera (14ta Edición). México: Pearson.

Gomes Novaes, P.; Neto, A.; de Almeida, A.; Louzada, L. (2015). Efeito clientela: uma análise da relação entre book-tax differences e dividendos extraordinários das empresas com ações listadas na Bovespa. Revista Contemporânea de Contabilidad, v. 12, n. 26, 3-22. http://dx.doi.org/10.5007/2175-8069.2015v12n26p3

Gordon, M. J. (1959). Dividends, earnings, and stock prices. The Review of Economics and Statistics, v. 41, n. 2, part 1, 99-105. https://doi.org/10.2307/1927792

Govindaraj, S.; Li, Y.; Zhao, C. (2020). The effect of option transaction costs on informed trading in the options market around earnings announcements. Journal of Business Finance \& Accounting, v. 47, n. 5-6, 615-644. https://doi.org/10.1111/jbfa.12443

Gutiérrez Urzúa, M.; Yañez Alvarado, M.; Umaña Hermosilla, B. (2012). Análisis de la política de pago de dividendos en empresas chilenas. Estudios Gerenciales, v. 28, n. 123, 27-42. https://doi.org/10.1016/S0123-5923(12)70203-6

Hernández-Chacín, A.; Hernández-Fernández, L.; Portillo-Medina, R.; Romero Borré, J. (2017). Decisiones sobre dividendos en hipermercados y supermercados familiares en el estado Zulia, Venezuela. Revista Espacios, v. 38, n. 23, 29.

Higgins, R. C. (1972). The corporate dividend-saving decision. The Journal of Financial and quantitative analysis, v. 7, n. 2, 1527-1541. https://doi.org/10.2307/2329932

Higuerey Gómez, A. (2014. Política de Dividendos. Universidad de los Andes. URL

Jensen, M. C. (1986). Agency costs of free cash flow, corporate finance, and takeovers. The American Economic Review, v. 76, n. 2, 323-329. URL

John, K.; Williams, J. (1985). Dividends, dilution and taxes: a signalling equilibrium. The Journal of Finance, v. 40, n. 4, 1053-1070. https://doi.org/10.2307/2328394

Kristjanpoller Rodríguez, W.; Silva Arroyo, J. (2014). El impacto de la propiedad institucional de las administradoras de fondos de pensión sobre la política de dividendos: evidencia para Chile. Cuadernos de Administración, v. 27, n. 49, 11-36. https://doi.org/10.11144/Javeriana.cao27-49.ipia 
Kumar, P. (1988). Shareholder-manager conflict and the information content of dividends. The Review of Financial Studies, v. 1, n. 2, 111-136. https://doi.org/10.1093/rfs/1.2.111

La Porta, R.; Lopez-de-Silanes, F.; Shleifer, A.; Vishny, R. W. (2000). Agency problems and dividend policies around the world. The Journal of Finance, v. 55, n. 1, 1-33. https://doi.org/10.1111/0022-1082.00199

Lefort, F. (2008). El efecto de los conflictos de agencia en las políticas de dividendos a los accionistas. El caso chileno. El Trimestre Económico, v. 75, n. 299, 597-639. https://doi.org/10.20430/ete.v75i299.413

Leite, M.; Bogoni, N. M.; Hein, N. (2018). Revisitando os determinantes da distribuição de dividendos: uma análise em empresas brasileiras. Revista Ambiente Contábil, v. 11, n. 1, 58-76. https://doi.org/10.21680/2176-9036.2019v11n1ID14152

Ley 6.404 de 1976. Ley de sociedades. 15 de diciembre de 1976. Presidencia de la Republica. Brasilia. $\underline{U R L}$

Ley 18.046 de 1981. Sobre Sociedades Anónimas. 22 de octubre de 1981. Junta de Gobierno de la República de Chile. URL

Ley 19.550 de 1984. Ley general de sociedades. 30 de marzo de 1984. Ministerio de Justicia y Derechos Humanos. Buenos Aires. URL

Lintner, J. (1956). Distribution of Incomes of corporations among dividends, Retained Earnings, and Taxes. The American Economic Review, v. 46, n. 2, 97-113. URL

Llorente Muñoz, V. (2012). Política de dividendos y conflicto en la empresa familiar: el protocolo. Revista de Empresa Familiar, v. 2, n. 1, 55-63. https://doi.org/10.24310/ejfbejfb.v2i1.4064

López Iturriaga, F. J.; Rodríguez Sanz, J. A. (1999). La decisión de dividendos en la empresa española: un contraste de teorías alternativas. Cuadernos de Economía y Dirección de la Empresa, n. 3, 2944. $\underline{U R L}$

López Iturriaga, F.; Saona Hoffmann, P. (2007) Endeudamiento, dividendos y estructura de propiedad como determinantes de los problemas de agencia en la gran empresa española. Cuadernos de Economía y Dirección de la Empresa, v. 10, n. 31, 119-146. https://doi.org/10.1016/S1138-5758(07)70085-3

Lozano, M. B.; De Miguel, A.; Pindado, J. (2002). Papel de la política de dividendos en las empresas reguladas. Investigaciones económicas, v. 26, n. 3, 447-474. URL

Makaew, T.; Maksimovic, V. (2020). Competition and operating volatilities around the world. Journal of Financial and Quantitative Analysis, v. 55, n. 2, 517-547. https://doi.org/10.1017/S002210901800159X 
Maquieira V., C.; Moncayo M., I. (2004). Costos de Agencia y Costos de Transacción como Determinantes de la tasa de pago de dividendos en Chile: Extensión. Estudios de Administración, v. 11, n. 2, 1-26. https://doi.org/10.5354/0719-0816.2004.56799

Maquieira V., C.; Osorio M., D. (2000). Anuncios de cambios en pagos de dividendos y su impacto en la riqueza de los accionistas: evidencia empírica en Chile. Estudios de Administración, v. 7, n. 1, 1-26. https://doi.org/10.5354/0719-0816.2000.56746

Mascareñas, J. (2011). La política de dividendos. https://dx.doi.org/10.2139/ssrn.2312666

Menezes da Silva, R. L.; Ciampaglia Nardi, P. C.; Tonani, R. (2016). A relevância do dividendo adicional proposto. Revista Contemporânea de Contabilidade, v. 13, n. 29, 179-202.

https://doi.org/10.5007/2175-8069.2016v13n29p179

Miller, M. H.; Modigliani, F. (1961). Dividend policy, growth, and the valuation of shares. Journal of Business, v. 34, n. 4, 411-433. URL

Miller, M. H.; Rock, K. (1985). Dividend policy under asymmetric information. The Journal of Finance, v. 40, n. 4, 1031-1051. https://doi.org/10.1111/j.1540-6261.1985.tb02362.x

Mongrut Montalvan, S.; Delfino Barilla, C.; Devercelli Ruiz, G.; Lambarri Figueroa, D. (2017). Política de dividendos y buen gobierno corporativo en el Perú: ¿Existe alguna relación? Revista Mexicana de Economía y Finanzas Nueva Época REMEF, 2017, v. 12, n. 2, 103-116. https://doi.org/10.21919/remef.v12i2.93

Núñez Pérez, J.; Rositas Martínez, J.; Medina Elizondo, M. (2013) Modelo de política óptima de dividendos. Innovaciones de Negocios, v. 10, n. 20, 251-273. URL

Pieloch-Babiarz, A. (2020). Characteristics identifying the companies conducting different dividend policy: evidence from Poland. Equilibrium. Quarterly Journal of Economics and Economic Policy, v. 15, n. 1, 63-85. https://doi.org/10.24136/eq.2020.004

Pinheiro, A.; Dias, A. C. (2012). Dividendos e efeito clientela: Evidencias no mercado brasileiro. Revista de Administracao de Empresas, v. 52, n. 4, 448-463. http://dx.doi.org/10.1590/S0034-75902012000400007

Rodrigues Sobrinho, W.; Simões Rodrigues, H.; Sarlo Neto, A. (2014). Dividendos e accruals discricionários: um estudo sobre a relação entre a política de distribuição de dividendos e a qualidade dos lucros. Revista Contemporânea de Contabilidade, v. 11, n. 24, 3-23. https://doi.org/10.5007/2175-8069.2014v11n24p3

Ross, S. A. (1977). The determination of financial structure: The incentive-signalling Approach. The Bell Journal of Economics, v. 8, n. 1, 23-40. https://doi.org/10.2307/3003485

Ross, S. A., Westerfield, R. W., Jaffe, J. F. (2012). Finanzas Corporativas. Mexico: McGraw-Hill. 
Ross, S. A.; Westerfield, R. W.; Jordan, B. D. (2010). Fundamentos de finanzas corporativas. México: McGraw-Hill.

Rozeff, M. S. (1982). Growth, beta and agency costs as determinants of dividend payout ratios. Journal of Financial Research, v. 5, n. 3, 249-259. https://doi.org/10.1111/j.1475-6803.1982.tb00299.x

Sánchez Gálvez, C. (2012). Determinantes de la política de dividendos en Chile: Años 2007-2010 (Tesis de pregrado). $\underline{U R L}$

San Martín Reyna, J. M. (2017). Estructura de propiedad y su efecto en la política de dividendos en el contexto mexicano. Contaduría y Administración, v. 62, n. 4, 1183-1198. https://doi.org/10.1016/i.cya.2015.12.005

Santos Jiménez, N. (2008). La política de dividendos y la rentabilidad de los negocios. Industrial Data, v. 11, n. 1, 29-36. https://doi.org/10.15381/idata.v11i1.6029

Smith, D. M. (2011). Residual dividend policy. En H. K. Baker (Ed.), Dividends and Dividend Policy (pp. 115-126). John Wiley \& Sons. https://doi.org/10.1002/9781118258408.ch7

Usma Patiño, J. A.; Ramírez Arango, C. M. (2010). Gobierno corporativo y política de dividendos: El caso colombiano (Tesis de maestría). $\underline{\mathrm{URL}}$

Vancin, D.; Laser Procianoy, J. (2016). Índices contábeis e a decisão do pagamento de dividendos. Revista Contemporânea de Contabilidade, v. 13, n. 28, 57-79. https://doi.org/10.5007/2175-8069.2016v13n28p57 